\title{
DIFUSIÓN DE PRODUCTOS A TRAVÉS DE REDES SOCIALES: UNA REVISIÓN BIBLIOGRÁFICA UTILIZANDO LA TEORÍA DE GRAFOS
}

\section{DIFFUSION OF PRODUCTS THROUGH SOCIAL NETWORKS: A LITERATURE REVIEW USING GRAPH THEORY}

\author{
Sebastián Robledo-Giraldo ${ }^{1} \quad$ Néstor Darío Duque-Méndez $z^{2}$ Jorge Iván Zuluaga-Giraldo
}

Forma de citar: ROBLEDO-GIRALDO Sebastián, DUQUE-MÉNDEZ Néstor, ZULUAGA-GIRALDO Jorge. Difusión de productos a través de redes sociales: una revisión bibliográfica utilizando la teoría de grafos. Respuestas. 2013; 18(2): 28-42.

Recibido:

Mayo 3 de 2013

Aceptado:

Septiembre 15 de 2013

\section{RESUMEN}

La difusión de productos a través de redes sociales es un campo de aplicación del mercadeo, donde la decisión de compra de un consumidor es influenciada por factores internos y externos como su red de conocidos y familiares. El propósito de esta investigación es identificar las principales perspectivas y plantear futuras investigaciones, apoyados en la revisión selectiva del estado del arte. Para la orientación de la búsqueda y la selección de artículos se utilizó la teoría de grafos, aprovechando las posibilidades de reconocer las conexiones entre los diferentes trabajos, arrojando para su análisis 18 artículos clásicos y 23 artículos actuales. A partir de esto se obtuvo, como resultado de la investigación, cuatro (4) estrategias de mercadeo diferentes: enfocadas a los influenciadores, a los no influenciadores, grupos pequeños y estrategias tradicionales de mercadeo.

Palabras clave: difusión de productos, redes sociales, teoría de grafos.

\section{ABSTRACT}

The diffusion of products through social networking is an application field of marketing, where the buying decision of a consumer is influenced by internal and external factors as their network of friends and relatives. The purpose of this research is to identify the main perspectives and propose future research, supported in state of the art selective review. As input for the orientation of search and articles selection, graph theory was used, leveraging the odds of recognizing the links among different works, providing for analysis 18 classic articles and 23 current articles. The result of the investigation showed four different marketing strategies: focused on influencers, non-influencers, small groups and traditional marketing strategies.

Keywords: diffusion of products, social networks, graph theory. 


\section{INTRODUCCIÓN}

$\mathrm{L}$ a difusión social de productos a través de redes sociales, es un tema que ha tenido en los últimos años un gran desarrollo, gracias a la disponibilidad de datos reales de Internet (Baker, 2009). Los primeros avances los realizaron Bass (1969) y Rogers (1962), aunque sus modelos suponían redes homogéneas, fue un gran aporte para su época. Ahora, la tendencia es analizar los procesos de difusión, teniendo en cuenta los conceptos iniciales, pero en redes heterogéneas (Peres et al. 2010). En este artículo se identifican las principales propuestas en esta área, a partir de una revisión bibliográfica selectiva.

Para realizar la búsqueda de los artículos pertinentes con el tema de investigación y que apoyaran el objetivo planteado, se utilizó la teoría de grafos, pues los resultados obtenidos mostraron la capacidad del enfoque para una valiosa separación de las referencias relevantes. Esta teoría permite ver los artículos como nodos y las referencias como enlaces y, a partir de esto aplicar diferentes estadísticas para encontrar los artículos "clásicos" y los artículos "actuales" más importantes. El resultado arrojó 41 artículos, 18 clásicos y 23 actuales.

Con esta selección se hizo el análisis orientado a identificar los diferentes enfoques de la difusión social de productos a través de redes sociales. Cuatro fueron las variantes en las visiones predominantes encontradas: la primera se concentró en las personas influenciadoras, la segunda en las no influenciadoras, la tercera en grupos pequeños y la cuarto en estrategias tradicionales de mercadeo.

El resto del artículo se divide de la siguiente manera: la sección dos explica el proceso de selección de artículos utilizando la teoría de grafos. La tercera sección explica los diferentes resultados encontrados, desde artículos clásicos, reviews y actuales. Por último, en la cuarta sección se plantea las conclusiones, limitaciones del estudio y futuras investigaciones.

\section{METODOLOGÍA}

Junio - Diciembre 2013

ISSN 0122-820X

En la presente investigación se aplicó la teoría de grafos (Euler, 1941) para identificar los artículos más pertinentes en el tema de difusión social de productos a través de redes sociales. La teoría de grafos ha tenido una gran aplicación en las ciencias sociales (Wasserman y Faust, 1994), donde se analizan y calculan las propiedades estructurales de estas redes para predecir el comportamiento de los nodos. Para poder aplicar esta teoría se consideró los artículos y sus diferentes referencias como una red de conocimiento, donde se busca identificar los principales artículos por medio de indicadores como el grado de entrada y salida de cada nodo.

Aunque el concepto de redes de conocimiento no se ha definido de forma clara aún (Liu et. al. 2013), se puede considerar que los nodos representan unidades de conocimiento (en este caso artículos) y los enlaces indican las conexiones entre estos artículos (en este caso las referencias que tienen los artículos). Un ejemplo se puede observar en la Figura 1.

Figura 1. Ejemplo de una red de conocimiento con indicadores de entrada y salida.

Los nodos son artículos y los enlaces son citaciones

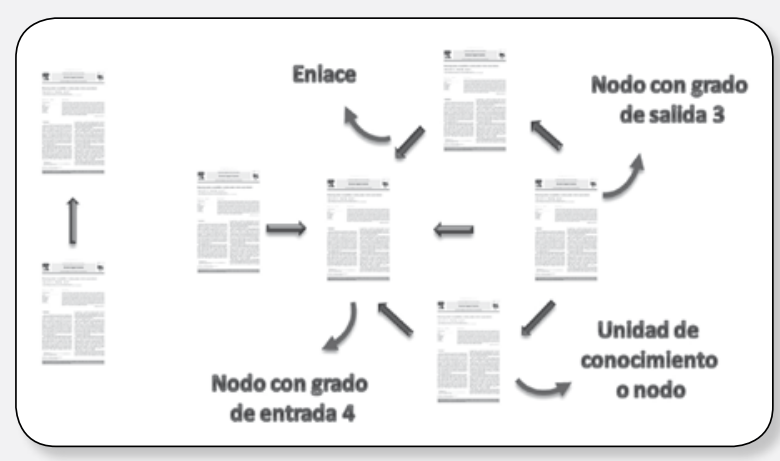

Por otro lado, la teoría de grafos se ha convertido en una herramienta ampliamente aceptada para revelar y cartografiar las estructuras de las redes sociales (Tonta y Darvish, 2010). En la presente investigación se van a considerar dos indicadores para seleccionar los artículos de mayor importancia. El primer indicador es el grado de entrada de cada nodo, este muestra la cantidad de artículos que están 
No. 2

Junio - Diciembre 2013 ISSN 0122-820X

PP: $28-42$ referenciando a uno en particular. El segundo indicador es el grado de salida, que muestra la cantidad de artículos que referencia un artículo dentro del área de conocimiento que se está investigando. Un ejemplo de estos indicadores se muestra en la Figura 1.

Una vez identificados los indicadores con los que se va a trabajar, se procedió a realizar la búsqueda en Web of Knowledge en la base de datos Thomson Reuters' Web of Science (WoS) dentro del periodo de tiempo de enero de 2001 hasta el 3 de Septiembre de 2013. Para identificar la literatura asociada se realizó la siguiente ecuación de búsqueda:

\section{Topic $=($ products $)$ AND Topic $=($ diffusion $)$ AND Topic $=($ social network $)$ \\ Refined by: Document Types $=($ ARTICLE $)$ Timespan $=$ All years. Databases $=$ SCI-EX- PANDED, SSCI, A\&HCI.}

Se obtuvieron un total de 100 artículos. Una vez descargada la información principal de los artículos (título, autores, abstract, palabras claves y referencias entre otras) se convirtió manualmente el archivo *.txt a *.isi para cargarlo en el software Sci2tool (Sci2 Team, 2009) y poder generar la red. Después se buscó, dentro de la red, datos de artículos duplicados y para esto se utilizó el algoritmo de Winkler (1990) con un 95\% de similitud. Un ejemplo del procedimiento se puede observar en la Figura 2.

Figura 2. Proceso para transformar la información de los artículos y sus referencias en una red o grafo.
Una vez se tiene la red, se exporta al software Gephi (Bastian et. al. 2009) para realizar los cálculos de los indicadores de grado de entrada y salida de cada artículo. El resultado, es una red de 4.186 nodos y 5.393 enlaces.

Posteriormente se determinó el grado de cada nodo, luego se eliminaron los nodos de grado de entrada 1 y grado de salida 0 , es decir, los artículos que fueron referenciados sólo una vez y nunca referenciaron a otros artículos de esta red; estos artículos se eliminaron porque pierden relevancia dentro del área de conocimiento en la que se está haciendo la búsqueda sistemática. En la Figura 3 se puede observar las diferentes redes que se generaron durante el proceso, hasta la red final.

Figura 3. Procedimiento para determinar el grado de entrada y salida de cada artículo

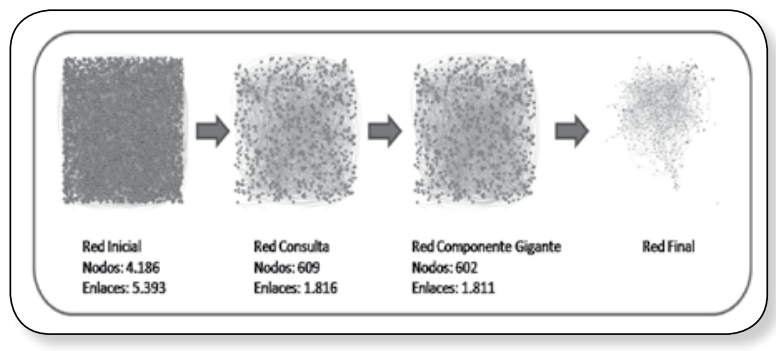

El resultado es una red con 609 nodos y 1.816 enlaces. A continuación se determinó el componente gigante que consiste en eliminar subredes o nodos que se encuentran aislados de la gran red de artículos que más han hecho aportes al área del conocimiento específica. El resultado del componente gigante es una red de 602 nodos y 1.811 enlaces.

Luego, se identificó dentro de ese gran componente los artículos relevantes de acuerdo a las estadísticas de grado de entrada y salida del nodo. En la Figura 4 se puede observar dos redes, en la primera se identificaron los artículos más relevantes de acuerdo al grado de entrada y en la segunda de acuerdo al grado de salida. 
Figura 4. Redes finales de acuerdo al grado. El tamaño de cada nodo depende del valor del grado.

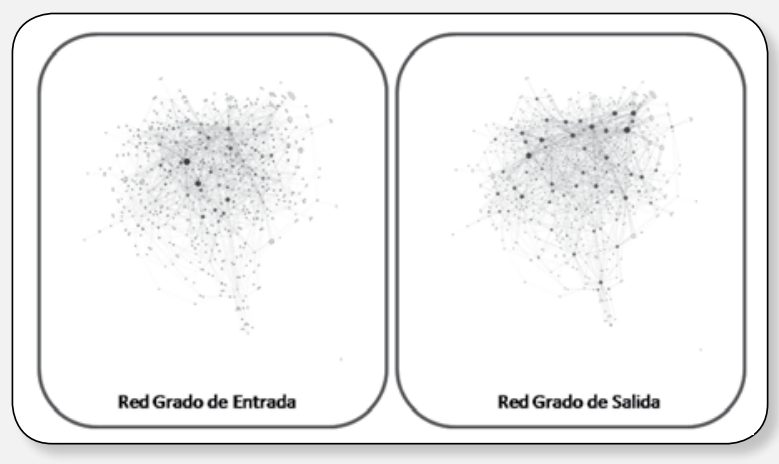

La red final tiene artículos, libros y reviews, por lo tanto, se excluyeron 6 libros y se dejó, por su importancia, la obra clásica de Rogers (2003). También, se separaron los 3 reviews. Por lo tanto, de cada una de estas redes (grado de entrada y de salida), se seleccionaron 18 artículos clásicos y 23 actuales. Con estos artículos se procedió a construir el marco teórico del tema "difusión de productos a través de redes sociales"

\section{ENFOQUES ENCONTRADOS}

Desde hace varios años se ha sabido que existe una influencia social en algunos procesos de decisión de las personas, como por ejemplo, el hábito de fumar (Christakis y Fowler, 2008), desertar del servicio militar (de Paula, 2009) o dejar el empleo (Castilla, 2005). Esto también se puede aplicar a los procesos de difusión a través de las estructuras o redes sociales (Jager y Janssen, 2001). El tema es importante para los mercadólogos, ya que con esta información pueden entender mejor los procesos de contagio social y crear estrategias más efectivas.

La difusión de productos a través de redes sociales se ha estudiado desde la perspectiva de la difusión de innovaciones (Rogers, 2003; Bass, 1969). La difusión social se puede definir como el proceso de penetración de mercado de nuevos productos o servicios, que es impulsada por la influencia social y estas influencias incluyen todas las interdependencias entre consumidores que afectan varios jugadores de mercado con o sin su conocimiento explícito
(Peres et al. 2010). Por lo tanto, la difusión de productos a través de redes sociales analiza cómo los diferentes actores influencian, de manera particular y global, la compra de un producto.

La difusión de innovaciones se puede dividir en 7 áreas: redes sociales, externalidades de la red, despegues y repiques, generaciones de tecnologías, influencia cruzada de países, crecimiento de diferencias a través de países y, por último, crecimiento y competencia (Peres et al. 2010). El trabajo presentado en este artículo se orienta a la difusión social de productos desde la perspectiva de las redes sociales. Una precisión importante para este estudio es que cuando se habla de redes sociales se hace referencia a las redes de amistad o parentesco reales y no a redes sociales virtuales como facebook o twitter, aunque para su estudio se pueda utilizar la información de estas redes sociales online.

Una red social es una estructura social compuesta por actores (en este caso individuos) que están conectados por enlaces, que se pueden interpretar como lazos de amistad o parentesco (Iribarren y Moro, 2011). Este tipo de relaciones, tanto las individuales como la estructura global de la red, influencian a los individuos (Fowler y Christakis, 2008). En conjunto, las redes sociales son la base en la que se mueve la difusión de productos.

El mercadeo basado en la red se refiere a un conjunto de técnicas de marketing que aprovechan los vínculos entre los consumidores para aumentar las ventas. Algunos ejemplos: el mercadeo boca a boca, la difusión social, buzz marketing y el mercadeo viral (Hill et al. 2006). ISSN 0122-820X PP: 28-42

Por otro lado, Van den Bulte y Wuyts (2007, 49-50) realizaron una clara diferencia entre buzz marketing y marketing viral. El buzz Marketing se puede considerar como un tipo de mercadeo boca a boca que no explota la estructura de la red y el objetivo es captar la atención de los medios y consumidores. En cambio el Marketing Viral se refiere más a las 
No. 2

Junio - Diciembre 2013 ISSN 0122-820X

PP: $28-42$ actividades de promoción boca a boca online, es decir, a la ayuda y cooperación entre los consumidores para transmitir los mensajes (Liu-Thompkins, 2012).

Por otra parte, es necesario analizar la dinámica y la estructura de la red en el proceso de difusión para poder entender los diferentes comportamientos que se presentan. Este tema se ha estudiado poco y la atención se ha centrado en la influencia de individuos centrales o hubs (Van den Bulte y Yoshi, 2007; Watts y Dodds, 2007). Es por esto, que el papel de la estructura y la dinámica de la red en la influencia de la difusión de productos está en su infancia (Peres et al. 2010).

Un aparte importante en el análisis lo constituye lo referente a los artículos de revisión encontrados, puesto que al momento de su publicación recogen los enfoques detectados por los autores. Mahajan et al. (1990) realizaron una revisión de los aportes más importantes entre 1969 y 1989, llegando a la conclusión que existían 4 sub-áreas: modelos básicos de difusión, consideraciones de estimación al parámetro, modelos de difusión flexible, extensiones y refinamientos, y el uso de modelos de difusión. Peres et al. (2010), propusieron 7 áreas de la difusión de innovaciones y recomendaron investigaciones más profundas en redes heterogéneas. Por otro lado, Libai et al. (2010), analizaron las diferentes interacciones entre consumidores online teniendo en cuenta 4 enfoques: las dimensiones $\mathrm{C} 2 \mathrm{C}$, los sistemas sociales $\mathrm{C} 2 \mathrm{C}$, contexto $\mathrm{C} 2 \mathrm{C}$ y la evaluación de los efectos C2C. Este artículo muestra la evolución del concepto de difusión de productos a través de redes sociales desde los primeros artículos hasta encontrar diferentes enfoques actuales, utilizando la teoría de grafos para identificarlos.

\subsection{Artículos Clásicos}

En esta subsección se tratarán los artículos con mayor grado de entrada en la búsqueda para el área de conocimiento: "difusión social de productos a través de redes sociales". La explicación de los artículos se realiza de una manera cronológica.
Tabla 1. Artículos clásicos de acuerdo al grado de entrada.

\begin{tabular}{|l|}
\hline Artículos Clásicos \\
\hline Bass (1969), Granovetter (1973, 1978 y 1985), Burt \\
(1987), Brown y Peter (1987), Watts y Strogatz \\
(1998), Barabási et al. (1999), Van den Bulte y \\
Lilien (2001), McPherson (2001), Goldenberg et \\
al. (2002), Rogers (2003), Van den Bulte y Stefan \\
(2004), Godes y Mayzlin (2004), García (2005), \\
Hill (2006), Watts y Dodds (2007), Van den Bulte y \\
Yoshi (2007) y Goldenberg et al. (2009). \\
\hline
\end{tabular}

La investigación sobre la difusión de productos nace con los trabajos de Rogers (1962) y Bass (1969) quienes analizaron el comportamiento de la difusión social de innovaciones. Rogers (1962) propuso 5 tipos diferentes de individuos dentro del proceso de difusión de innovaciones: innovadores, primeros seguidores, mayoría precoz, mayoría tardía y rezagada. Bass (1969) propuso un modelo similar con otro tipo de individuos: los imitadores e innovadores.

Las dos propuestas se basaron en modelos homogéneos, donde la cantidad de conexiones entre individuos eran iguales y no se tenían en cuenta las interacciones entre individuos. Aunque, estos modelos presentan ventajas como: la facilidad para realizar pronósticos y la poca intuición necesaria para definir el efecto de la relaciones individuales a nivel global, en la realidad, las redes sociales, no son homogéneas y sus interacciones son complejas (Kossinets y Watts, 2006).

Unos años más tarde se dio un avance importante en el conocimiento de las dinámicas que ocurren dentro de la red social que son importantes para los procesos de difusión, como: el puente que hacen los enlaces débiles y su importancia para el flujo de la información (Granovetter, 1973) que más adelante confirmaron Brown y Reingen (1987), la influencia de la red social en los procesos de decisión de una persona (Granovetter, 1978 y 1985), la importancia de los huecos estructurales (Burt, 1987) y, por último, McPherson et al. (2001), explicaron desde una perspectiva de las redes sociales, el concepto de homofilia. 
Después, aparecieron dos grandes adelantos gracias a la posibilidad de extraer datos de Internet y a la posibilidad de acceder a diferentes tipos de información. Los primeros fueron Watts y Strogatz (1998), con la explicación de los seis grados de separación por medio del mundo pequeño. Los segundos, Barabási et. al. (1999) que descubrieron una topología especial de la red. Explicaron cómo diferentes tipos redes podrían tener el mismo comportamiento de una red de escala libre con atracción preferencial. Estos dos descubrimientos permitieron ver las redes de forma heterogénea, con la posibilidad de realizar predicciones y entender mejor qué pasaba durante las interacciones de los diferentes individuos que participaban dentro del proceso de difusión.

Aunque se han realizado algunas críticas a los modelos de difusión de productos o innovaciones (Van den Bulte y Lilien, 2001; Van den Bulte y Stremersch, 2004), después de los hallazgos en redes sociales online, esta área de conocimiento ha tenido un gran crecimiento. Algunas de las técnicas para simular estos procesos han sido las células autómatas (Goldenberg et al. 2002) y modelado basado en agentes (García, 2005).
En adelante, las investigaciones se han enfocado en establecer cuáles son los mecanismos que facilitan o inhiben los procesos de difusión. Esto ha llevado a explicaciones desde las personas altamente influenciadoras (Goldenberg et al. 2009), la estructura de la red (Hill et al. 2006; Van den Bulte y Yoshi, 2007) hasta encontrar una masa crítica que comience ese crecimiento acelerado de la adopción de un producto (Watts y Dodds, 2007). Sin embargo, existen varias dificultades para realizar estas investigaciones como la adquisición de los datos, identificar cuáles de esos datos son importantes y generar la gráfica de la red (Godes y Mayzlin, 2004).

\subsection{Enfoques}

Dentro de la búsqueda se encontraron diferentes perspectivas del tema de "difusión de productos a través de redes sociales". Estas perspectivas van desde proponer estrategias de mercadeo para mejorar el proceso de difusión con influenciadores, grupos pequeños y estrategias tradicionales hasta modelos de producción, proyección de ventas y adopción. Un resumen se puede observar en la Tabla 2.

Tabla 2. Artículos actuales de acuerdo al grado de salida. Diferentes enfoques acerca de la difusión de productos a través de redes sociales.

\begin{tabular}{|c|c|c|c|c|}
\hline Estudios & Producto & Perspectivas & Técnica & Conclusión \\
\hline $\begin{array}{l}\text { Laciana et } \\
\text { al. (2013) }\end{array}$ & - & $\begin{array}{c}\text { Estrategias } \\
\text { tradicionales de } \\
\text { mercadeo }\end{array}$ & $\begin{array}{c}\text { Modelado Basado } \\
\text { en Agentes }\end{array}$ & $\begin{array}{c}\text { Bajar el precio del } \\
\text { producto al inicio, invertir } \\
\text { mucho en publicidad al } \\
\text { principio, utilizar las } \\
\text { diferentes tecnologías } \\
\text { sociales disponibles. }\end{array}$ \\
\hline $\begin{array}{l}\text { Libai et al. } \\
\qquad(2013)\end{array}$ & $\begin{array}{l}12 \text { Redes } \\
\text { reales }\end{array}$ & $\begin{array}{c}\text { Estrategias } \\
\text { tradicionales de } \\
\text { mercadeo }\end{array}$ & $\begin{array}{l}\text { Modelado Basado } \\
\text { en Agentes - } \\
\text { Redes Reales }\end{array}$ & $\begin{array}{c}\text { Muestran como la } \\
\text { aceleración y la expansión } \\
\text { generan el valor de un } \\
\text { programa }\end{array}$ \\
\hline $\begin{array}{l}\text { Choi et al. } \\
\text { (2010) }\end{array}$ & - & $\begin{array}{c}\text { Grupos } \\
\text { Pequeños }\end{array}$ & \begin{tabular}{|} 
Modelo \\
computacional de \\
redes sociales - \\
Mundo Pequeño
\end{tabular} & $\begin{array}{l}\text { Enfocarse en los puentes } \\
\text { que hay entre grupos y en } \\
\text { pequeños grupos densos }\end{array}$ \\
\hline
\end{tabular}


No. 2
Tabla 2. Artículos actuales de acuerdo al grado de salida. Diferentes enfoques acerca de la difusión de productos a través de redes sociales.

\begin{tabular}{|c|c|c|c|c|}
\hline Estudios & Producto & Perspectivas & Técnica & Conclusión \\
\hline $\begin{array}{l}\text { Goldenberg } \\
\text { et al. (2007) }\end{array}$ & - & $\begin{array}{l}\text { Grupos } \\
\text { Pequeños }\end{array}$ & $\begin{array}{c}\text { Modelado Basado } \\
\text { en Agentes }\end{array}$ & $\begin{array}{l}\text { Pasa lo contrario de Choi } \\
\text { et al. (2010). La pequeña } \\
\text { masa de personas que } \\
\text { hacen comentarios } \\
\text { negativos se propaga muy } \\
\text { rápido. También los } \\
\text { puentes ayudan a } \\
\text { potencializar estos } \\
\text { comentarios negativos. }\end{array}$ \\
\hline \begin{tabular}{|c|} 
Bohlmann \\
et al. (2010)
\end{tabular} & - & $\begin{array}{l}\text { Grupos } \\
\text { Pequeños }\end{array}$ & $\begin{array}{c}\text { Modelado Basado } \\
\text { en Agentes }\end{array}$ & $\begin{array}{l}\text { Redes con alto grado de } \\
\text { clusterización }\end{array}$ \\
\hline $\begin{array}{l}\text { Katona et } \\
\text { al. (2010) }\end{array}$ & $\begin{array}{l}\text { Invitación a } \\
\text { una red social }\end{array}$ & $\begin{array}{c}\text { Grupos } \\
\text { Pequeños }\end{array}$ & $\begin{array}{c}\text { Empírico - Modelo } \\
\text { Hazard }\end{array}$ & $\begin{array}{l}\text { Enfocarse en grupos } \\
\text { pequeños y densos }\end{array}$ \\
\hline $\begin{array}{c}\text { Pegoretti } \\
\text { et al. (2012) }\end{array}$ & - & $\begin{array}{l}\text { Grupos } \\
\text { Pequeños }\end{array}$ & 4 No influyentes & $\begin{array}{c}\text { Concentrarse en los } \\
\text { grupos densos y los } \\
\text { puentes }\end{array}$ \\
\hline $\begin{array}{l}\text { Cho et al. } \\
\text { (2012) }\end{array}$ & - & $\begin{array}{l}\text { Grupos } \\
\text { Pequeños }\end{array}$ & $\begin{array}{c}\text { Modelo } \\
\text { estocástico } \\
\text { autómata celular }\end{array}$ & $\begin{array}{l}\text { La característica más } \\
\text { importante para una } \\
\text { rápida difusión es la } \\
\text { Centralidad social }\end{array}$ \\
\hline $\begin{array}{c}\text { Delre et al. } \\
(2007)\end{array}$ & - & $\begin{array}{l}\text { Grupos } \\
\text { Pequeños }\end{array}$ & $\begin{array}{c}\text { Modelado Basado } \\
\text { en Agentes }\end{array}$ & $\begin{array}{c}\text { La sincronización y } \\
\text { focalización de las } \\
\text { promociones afecta el } \\
\text { proceso de difusión social }\end{array}$ \\
\hline $\begin{array}{c}\text { Nitzan y } \\
\text { Libai (2011) }\end{array}$ & $\begin{array}{l}\text { Servicios de } \\
\text { telefonía } \\
\text { celular }\end{array}$ & Influenciadores & $\begin{array}{c}\text { Modelo de riesgo } \\
\text { proporcional }\end{array}$ & $\begin{array}{c}\text { Los clientes más } \\
\text { expuestos a la deserción } \\
\text { de un producto son los } \\
\text { altamente conectados y } \\
\text { los menos afectados son } \\
\text { los clientes leales }\end{array}$ \\
\hline $\begin{array}{l}\text { Yansong } \\
\text { (2013) }\end{array}$ & $\begin{array}{l}\text { Herramienta } \\
\text { tecnológica } \\
\text { para la } \\
\text { investigación } \\
\text { (mutegénesis) }\end{array}$ & Influenciadores & $\begin{array}{c}\text { Análisis de Redes } \\
\text { Sociales (ARS) }\end{array}$ & $\begin{array}{c}\text { El uso intensivo del } \\
\text { producto, la variedad en } \\
\text { los comportamientos de } \\
\text { uso y los vínculos } \\
\text { internacionales de las } \\
\text { personas altamente } \\
\text { conectadas, aumentan la } \\
\text { tasa de difusión } \\
\end{array}$ \\
\hline \begin{tabular}{|l} 
Haenlein y \\
Libai (2013)
\end{tabular} & $\begin{array}{l}\text { Planes de } \\
\text { Celulares }\end{array}$ & Influenciadores & $\begin{array}{c}\text { Experimento real } \\
\text { Modelado Basado } \\
\text { en Agentes }\end{array}$ & $\begin{array}{l}\text { Lograron una estrategia de } \\
\text { siembre óptima con los } \\
\text { líderes de ingresos con } \\
\text { sólo una siembra del 1\% }\end{array}$ \\
\hline
\end{tabular}


Tabla 2. Artículos actuales de acuerdo al grado de salida. Diferentes enfoques acerca de la difusión de productos a través de redes sociales.

\begin{tabular}{|c|c|c|c|c|}
\hline Estudios & Producto & Perspectivas & Técnica & Conclusión \\
\hline $\begin{array}{c}\text { Liu } \\
\text { Thompkins } \\
\text { (2013) }\end{array}$ & $\begin{array}{l}\text { Videos de } \\
\text { YouTube }\end{array}$ & Influenciadores & $\begin{array}{l}\text { Experimento real- } \\
\text { Modelo de Hazard } \\
\text { Proporcional }\end{array}$ & $\begin{array}{c}\text { Elegir lazos fuertes con el } \\
\text { promotor, } \\
\text { heterogeneidad entre los } \\
\text { consumidores elegidos y } \\
\text { la calidad del mensaje }\end{array}$ \\
\hline $\begin{array}{l}\text { Hinz et al. } \\
\text { (2013) }\end{array}$ & $\begin{array}{l}\text { Planes de } \\
\text { celulares }\end{array}$ & Influenciadores & $\begin{array}{c}\text { Experimento Real } \\
\text { Modelo } \\
\text { matemático }\end{array}$ & $\begin{array}{c}\text { Una estrategia de siembra } \\
\text { a los individuos mejor } \\
\text { conectadospuede ser } \\
\text { hasta } 8 \text { veces mejor }\end{array}$ \\
\hline $\begin{array}{l}\text { lyengar et } \\
\text { al. (2011) }\end{array}$ & Antiviral & Influenciadores & $\begin{array}{l}\text { Modelo Hazard - } \\
\text { Encuestas }\end{array}$ & $\begin{array}{c}\text { Elegir a las personas } \\
\text { altamente conectadas, de } \\
\text { acuerdo a un criterio } \\
\text { sociométrico, mejora el } \\
\text { proceso de difusión }\end{array}$ \\
\hline $\begin{array}{c}\text { Kratzer y } \\
\text { Lettl (2009) }\end{array}$ & - & Influenciadores & $\begin{array}{l}\text { ARS - Modelo } \\
\text { Jerárquico Lineal }\end{array}$ & $\begin{array}{c}\text { Es mejor enfocarse en } \\
\text { usuarios líderes que en } \\
\text { líderes de opinión, porque } \\
\text { los primeros, crean los } \\
\text { puentes entre diferentes } \\
\text { subredes } \\
\end{array}$ \\
\hline $\begin{array}{l}\text { Delre et al. } \\
\text { (2010) }\end{array}$ & - & Influenciadores & $\begin{array}{c}\text { Modelado Basado } \\
\text { en Agentes }\end{array}$ & $\begin{array}{l}\text { La importancia del } \\
\text { influenciador no radica en } \\
\text { su capacidad de convencer } \\
\text { sino en su capacidad de } \\
\text { multiplicar y difundir la } \\
\text { informaciónentre muchas } \\
\text { personas. }\end{array}$ \\
\hline $\begin{array}{l}\text { Liu } \\
\text { Thompkins } \\
\text { y Rogerson } \\
\text { (2012) }\end{array}$ & $\begin{array}{l}\text { Videos de } \\
\text { YouTube }\end{array}$ & No influyentes & $\begin{array}{l}\text { Proportional } \\
\text { Rates / Means } \\
\text { Models for } \\
\text { Recurrent Events }\end{array}$ & $\begin{array}{l}\text { Es mejor tener muchos } \\
\text { suscriptores con pocos } \\
\text { amigos que pocos } \\
\text { sucriptores con muchos } \\
\text { amigos. }\end{array}$ \\
\hline $\begin{array}{l}\text { Ceci y } \\
\text { Lubatti } \\
(2012)\end{array}$ & $\begin{array}{l}\text { Análisis } \\
\text { Empírico de } \\
\text { Pymes }\end{array}$ & $\begin{array}{l}\text { Desarrollo de } \\
\text { valores }\end{array}$ & $\begin{array}{l}\text { Cualitativa - } \\
\text { Análisis de } \\
\text { contenido }\end{array}$ & $\begin{array}{c}\text { La difusión de } \\
\text { innovaciones se mejora } \\
\text { por las relaciones } \\
\text { personales, la presencia } \\
\text { de la verdad, valores } \\
\text { compartidos y objetivos } \\
\text { comunes }\end{array}$ \\
\hline
\end{tabular}


No. 2

Junio - Diciembre 2013 ISSN 0122-820X

PP: $28-42$
Tabla 2. Artículos actuales de acuerdo al grado de salida. Diferentes enfoques acerca de la difusión de productos a través de redes sociales.

\begin{tabular}{|c|c|c|c|c|}
\hline Estudios & Producto & Perspectivas & Técnica & Conclusión \\
\hline $\begin{array}{l}\text { Goldenberg } \\
\text { et al. (2010) }\end{array}$ & \begin{tabular}{|} 
Máquinas de \\
Fax, radios $C B$, \\
lectores de \\
CD, lectores \\
de DVD y \\
servicios \\
celulares \\
\end{tabular} & $\begin{array}{l}\text { Efecto negativo } \\
\text { de la estructura }\end{array}$ & $\begin{array}{c}\text { Modelado Basado } \\
\text { en Agentes - } \\
\text { Casos empíricos }\end{array}$ & $\begin{array}{l}\text { Las externalidades de la } \\
\text { red crean un despegue } \\
\text { inicial despacio porque los } \\
\text { clientes potenciales } \\
\text { esperan por los } \\
\text { adoptantes tempranos }\end{array}$ \\
\hline $\begin{array}{l}\text { Fang et al. } \\
\text { (2013) }\end{array}$ & $\begin{array}{c}\text { Servicio de } \\
\text { telefonía } \\
\text { celular y Juego } \\
\text { Virtual }\end{array}$ & $\begin{array}{c}\text { Modelo de } \\
\text { probabilidad de } \\
\text { Adopción }\end{array}$ & $\begin{array}{c}\text { Método Bayesian } \\
\text { learning }\end{array}$ & $\begin{array}{l}\text { Los hallazgos sugieren que } \\
\text { la decisión de adopción en } \\
\text { una red social podrían ser } \\
\text { influenciados por varias } \\
\text { fuerzas relacionadas pero } \\
\text { distintas, mucho más allá } \\
\text { de la influencia social, y la } \\
\text { probabilidad de adopción } \\
\text { podría ser analizada y } \\
\text { predicha mejor desde una } \\
\text { perspectiva holística que } \\
\text { use más datos } \\
\text { comprensivos en redes } \\
\text { sociales }\end{array}$ \\
\hline $\begin{array}{c}\text { Amini et al. } \\
\text { (2012) }\end{array}$ & - & $\begin{array}{l}\text { Modelo de } \\
\text { producción }\end{array}$ & $\begin{array}{c}\text { Modelado Basado } \\
\text { en Agentes }\end{array}$ & $\begin{array}{c}\text { Determinaron la mejor } \\
\text { estrategia de producción - } \\
\text { ventas de un nuevo } \\
\text { producto genérico }\end{array}$ \\
\hline $\begin{array}{l}\text { Goldenberg } \\
\text { et al. (2009) }\end{array}$ & Software & $\begin{array}{l}\text { Modelo de } \\
\text { proyección de } \\
\text { ventas }\end{array}$ & $\begin{array}{c}\text { Modelos } \\
\text { estadísticos }\end{array}$ & $\begin{array}{c}\text { Se propone una } \\
\text { plataforma de modelado } \\
\text { universal que puede } \\
\text { capturar la naturaleza } \\
\text { estocástica de la dinámica } \\
\text { de ventas que al mismo } \\
\text { tiempo permite la } \\
\text { agregación de la conducta } \\
\text { individual }\end{array}$ \\
\hline
\end{tabular}

36 Durante la última década se han realizado diferentes investigaciones en el campo de la difusión de productos a través de redes sociales. Sin embargo, ha existido la dificultad de analizar el desempeño de las estrategias en el mundo real. Algunas propuestas han partido de estudios en estudiantes de colegio (Kratzer y Lettl, 2009), de videos en youtube (LiuThompkins, 2012), de simulaciones con agentes (Hanlein y Libai, 2013), del estudio de la difusión de una tecnología entre investigadores (Yansong, 2013), del mundo real como la difusión de un servicio de celulares (Hinz et al. 2012) y la difusión de un medicamento entre médicos (Iyengar et al. 2011).

\subsubsection{Estrategias de mercadeo basadas en} grupos pequeños y estrategias tradicionales Identificar las mejores formas de generar la difusión de un producto a través de redes sociales ha sido un reto para los mercadólogos. Este tipo de retos se ha intentado solucionar trabajando desde los influenciadores, no influenciadores, los grupos pequeños y estrate- 
gias tradicionales de mercadeo. Esta parte del artículo se concentra en las investigaciones que se han enfocado en utilizar la estructura de la red como facilitadora de los procesos de difusión.

Dentro de los procesos de difusión se ha investigado tanto lo que inhibe como lo que mejora la difusión. En estas investigaciones se han encontrado que la estructura de la red social puede acelerar o desacelerar el proceso de difusión. Goldenberg et al. (2007) analizaron el impacto de los comentarios negativos dentro de la masa crítica, los resultados fueron que, este tipo de comentarios, pueden generar un efecto devastador en el proceso de difusión y los enlaces débiles aceleran este proceso. Otro efecto negativo de la estructura de la red, es el efecto que tienen las externalidades en el crecimiento del mercado de un nuevo producto porque genera una desaceleración mientras los adoptantes tempranos crean el entorno para el crecimiento del producto (Goldenberg et al. 2010).

Para el estudio del proceso de difusión, se han propuesto varias perspectivas: el modelado matemático (Fang et al. 2013), modelado basado en agentes (Laciana et al. 2013) y modelos estadísticos (Goldenberg et al. 2009). Sin embargo, el modelado basado en agentes permite representar tanto comportamientos micros como macros de los diferentes agentes (García, 2005). También, el proceso de difusión se puede analizar de una forma inversa, desde la deserción de clientes, por ejemplo, Nitzan y Libai (2011) determinaron que existe una probabilidad del $80 \%$ que un cliente deje de comprar un producto cuando se expone a un cliente desertor.

Por otro lado, existe un consenso acerca de la importancia de la estructura de las redes en el proceso de difusión de un producto. Un ejemplo de la influencia que tienen los vecinos en la adopción de un nuevo producto, lo demuestra Katona et al. (2010), donde modelaron la decisión de adopción como un proceso de selección binaria y determinaron que los vecinos adoptantes y la densidad de la red tienen un efecto positivo en la decisión de adopción de un individuo. También la estructura de la red juega un papel importante en la velocidad de la difusión de un producto, porque la fortaleza de los vínculos entre innovadores y seguidores, permite activar las explosiones en cascada o los despegues de la difusión de productos (Katona et al. 2010). Por lo tanto, es necesario entender el efecto de la estructura para mejorar la difusión de un producto (Pegoretti et al. 2012; Liu-Thomkins y Rogerson, 2012).

El estudio del efecto de la estructura de la red con relación a la difusión de productos también se ha extrapolado a otras áreas. Las redes sociales juegan un papel importante en el éxito de los pequeños empresarios. La coexistencia de relaciones personales y profesionales forma un contexto único que altera la dinámica de la difusión (Ceci y Lubatti, 2012). También dentro del proceso de manufactura, la estructura de las redes de los compradores afecta el desempeño de la producción (Amini et al., 2012).

\subsubsection{Estrategias de mercadeo basadas en las personas más influyentes}

Dentro de la difusión social de productos se encontró otra perspectiva y es la que tradicionalmente se ha utilizado para realizar estrategias de mercadeo. Esta estrategia se enfoca en las personas que poseen en su red social una gran cantidad de contactos. Dentro de la literatura son conocidos como "hubs", líderes de opinión, usuarios líderes o líderes de ingresos. Existen investigaciones que han establecido diferentes estrategias para originar un mercadeo boca a boca a través de ellos, pero aún no existe un consenso de cuál sería la mejor.

Aunque en esta sección las estrategias se enfocan hacia individuos en particular en la red, diferentes investigaciones muestran que la proporción, su posición en la red y la capacidad de encontrarlos son elementos importantes para identificarlos. Por ejemplo, Kratzer y Lett (2009) definen dos individuos diferentes: usuarios líderes y líderes de opinión. Los usuarios líderes los caracterizaron porque tienen
Junio - Diciembre 2013

ISSN 0122-820X

PP: 28-42 
No. 2

Junio - Diciembre 2013 ISSN 0122-820X

PP: 28-42 un alto grado de intermediación y podían llegar a diferentes grupos de personas. Los líderes de opinión, por otro lado, se caracterizaban por tener un alto grado de centralidad, lo que los hace ser más influenciadores a su círculo social. Al final, los usuarios líderes resultaron ser la mejor propuesta para difundir un producto, ya que podían saltar entre diferentes grupos sociales y los líderes de opinión, aunque influenciaban fácilmente a su círculo social, este resultaba ser pequeño por las mismas restricciones de costo del líder de opinión (Barabasi y Bianconi, 2001), lo que confirma Liu-Thompkins (2012).

En oposición a estos resultados, se encuentra la investigación de Iyengar et al (2011). Ellos mencionaban la necesidad de desarrollar propuestas con datos reales, por lo tanto, su investigación se basó en el proceso de difusión de una medicina a través de médicos en una campaña real de mercadeo. Los resultados arrojaron que la estrategia de siembra más efectiva era con los médicos que tenían un alto grado de centralidad. Sin embargo, hacen la aclaración que esta definición de centralidad se debe hacer con base en la posición en la red y no por la percepción de los mismos clientes.

También Hinz et al. (2012), alude por más investigaciones basadas en datos reales. Ellos realizaron 3 experimentos de difusión: los dos primeros con una plataforma controlada y un video como producto, el tercero los realizaron con una empresa de celulares. El resultado fue que una estrategia de siembra a clientes más conectados "hubs" es 8 veces más efectiva que una orientada a seleccionar clientes aleatoriamente. Sin embargo, una efectividad aproximada dio una estrategia orientada a clientes con alta intermediación pero eligieron los que tuvieran mayor cantidad de conexiones por la facilidad de seleccionar este tipo de clientes, ya que para identificar los clientes con alta intermediación se necesita conocer todos los nodos y enlaces de la red.

Otra propuesta identifica los influenciadores como apalancadores de la difusión, como lo menciona Liu-Thompkins (2012), quien realizó un experimento real pero con videos de YouTube. Ella determinó las claves del éxito para una campaña de marketing viral con estrategias de siembra. Concluye además que una estrategia de siembra depende de la calidad del mensaje, de la conexión de los usuarios con el promotor del mensaje y de que exista una homogeneidad de intereses entre los usuarios elegidos. Sin embargo, consideró que los usuarios altamente conectados llevan a tener enlaces débiles y a disminuir la velocidad de difusión.

Una de las dificultades para las personas encargadas de realizar las campañas de mercadeo boca a boca, es seleccionar los clientes altamente conectados o los clientes con alta intermediación. Haenlein y Libai (2013) estudiaron el proceso de difusión, tanto en redes sociales reales como simuladas. Definieron a líderes de opinión y líderes de ingreso para determinar la estrategia de siembra más efectiva. Estudiaron los líderes de ingresos de la empresa por la facilidad de identificarlos y concluyeron que una siembra óptima fue de solo el $1 \%$ para este tipo de clientes.

Por último, Yansong (2013) sugirió que el uso intensivo y la alta variedad del comportamiento de uso tenían una relación positiva con la tasa de difusión. Estas dos características deben ser propias de los influenciadores para que se pueda dar un proceso de difusión. Por lo tanto, no basta con identificar influenciadores sino que deben tener características especiales. Para resumir, existe la necesidad de realizar investigaciones con datos reales sobre la difusión de productos a través de redes sociales (Libai et al, 2013 y Goldenberg et al., 2007) y que sus conclusiones se repitan en otros experimentos. También la definición de "influenciador" puede variar de acuerdo a las características de su red (Kratzer y Lettl, 2009) como por las características personales (Yansong, 2013).

\subsubsection{Pequeña masa crítica}

En la curva de difusión planteada por Rogers 
(1962), se encuentra un elemento de gran relevancia al momento de medir el impacto de un proceso de difusión, el cual se identifica como el despegue de la adopción. Es en este momento en el que comienza la penetración del mercado y se posiciona el producto en los consumidores. En los enfoques estudiados anteriormente, la discusión se centra en los iniciadores del proceso (influenciadores) y en las características del entorno (estructura de la red) como los elementos conductores de proceso de difusión. Para este tercer enfoque, los autores dirigen su atención a la masa crítica.

La masa crítica se define como el número suficiente de adoptantes de una innovación en un sistema social a partir del cual la tasa de adopción se vuelve auto sostenible y crea un mayor crecimiento (Rogers, 2003). En este orden de ideas, los esfuerzos intensivos en marketing dirigidos a la difusión del producto en el mercado son relevantes hasta cuando se alcanza la adopción de la masa crítica. De ahí en adelante, la misma red se encarga de propagar la adopción del nuevo producto como en un efecto de bola de nieve que permite identificar el preciso momento en que un producto despega (Delre et al., 2007). Ahora los esfuerzos de marketing deben ir enfocados al posicionamiento de marcas y estrategias de comercialización.

Desde este enfoque, los autores reconocen diferentes medios para alcanzar la masa crítica: Los influenciadores (Cho et al., 2012) y la óptima focalización y sincronización de los programas de promoción o marketing (Delre et al., 2007). Cho et al., (2012) definen las características de los influenciadores y cómo éstas se ven afectadas por las características de la red, identificando el tipo de influenciador adecuado para comenzar un programa de siembra según el tipo de innovación. Finalmente concluye que el rol del influenciador es importante hasta alcanzar la masa crítica. Por su parte, Delre et al. (2010) determina los tipos de grupos sobre los que se deben aplicar los programas de siembra, el momento y tiempo necesarios para llegar a la masa crítica. Encontraron que la estrategia óptima depen- de del tipo de innovación que se va a lanzar al mercado.

Junio - Diciembre 2013

ISSN 0122-820X

PP: 28-42

\section{CONCLUSIONES}

En este trabajo se analizaron los principales aportes al área de conocimiento "Difusión de productos a través de redes sociales". Se utilizó la teoría de grafos para identificar los artículos más importantes, según la ecuación de búsqueda aplicada en una base de datos de artículos especializados indexados. Esta metodología permitió encontrar artículos referentes al tema partiendo de las citaciones que existen entre ellos, identificando artículos clásicos y actuales con un alto porcentaje de efectividad.

En cuanto al tema de difusión social de productos a través de redes sociales, se encontraron diferentes perspectivas que van desde proponer estrategias de mercadeo para mejorar el proceso de difusión con influenciadores, grupos pequeños y estrategias tradicionales hasta modelos de producción, proyección de ventas y de adopción.

Aunque la metodología de simulación basada en agentes ha tenido una tendencia marcada para explicar o entender estas perspectivas porque permiten entender cómo las acciones en niveles micro afectan los comportamientos macros (Garcia, 2005), es necesario comenzar a realizar investigaciones de tipo empírico y que sus resultados se repitan en diferentes escenarios para consolidar una teoría acerca del tema, porque, como se pudo observar no existe un consenso acerca de la mejor estrategia de siembra para comercializar un producto (Hinz et al., 2012). Por lo tanto, es necesario realizar modelos más realistas y en un contexto social más amplio, considerando más elementos de las redes sociales como la influencia de la egonet o red personal y la influencia de la red online. También es necesario incluir diferentes características del target como el nivel socio-económico. También, futuras investigaciones deberían contemplar otras formas de difusión de productos que utilizan las estruc- 
No. 2

Junio - Diciembre 2013 ISSN 0122-820X

PP: 28-42 turas sociales como el marketing multinivel (Cruz y Olaya, 2008) para potencializar estas formas de comercialización y aplicarlos a otro tipos de empresas como las micro y pequeña (Robledo-Giraldo y Osorio-Zuluaga, 2012).

Por último, es importante que los investigadores propongan respuestas prácticas a los retos del área de "difusión de productos a través de estructuras sociales" para que puedan ser utilizadas por las personas encargadas del mercadeo, porque definir y encontrar personas "influenciadoras" o "grupos altamente conectados" puede ser una tarea compleja desde el punto de vista del análisis de redes sociales, como lo argumentan Haenlein y Libai, (2013).

\section{REFERENCIAS}

Amini, M., Wakolbinger, T., Racer, M., y Nejad, M. G. (2012). Alternative supply chain production-sales policies for new product diffusion: An agent-based modeling and simulation approach. European Journal of Operational Research, 216(2), 301-311. doi:10.1016/j. ejor.2011.07.040

Baker, S. (2009). What's a Friend Worth? Business Week, January (6), 32-36.

Barabási, A.-L., y Jeong, R. A. H. (1999). Mean-field theory for scale-free random networks.Statistical Mechanics and its Applications, 272(1), 173-187.

Bass, F. M. (1969). A New Product Growth for Model Consumer Durables.Management Science, 15(5), 215-227. doi:10.1287/ mnsc. 1040.0264

Bastian, M., Heymann, S., y Jacomy, M. (2009).Gephi: an open source software for exploring and manipulating networks. International AAAI Conference on Weblogs and Social Media.

Bianconi, G., y Barabási, A.-L.(2001). Competition and multiscaling in evolving networks.Europhysics Letters (EPL), 54(4), 436442. doi:10.1209/epl/i2001-00260-6

Brown, J. J., y Reingen, P. H. (1987). Social

ties and Word-of-Mouth referral behavior. Journal of Consumer Research, 14(3), 350-362.

Bulte, C. Van den, y Wuyts, S. (2007). Social Networks and Marketing. Cambridge, MA: Marketing Science Institute.

Burt, R. S. (1987). Social contagion and innovation: Cohesion versus structural equivalence. American journal of Sociology, 92 (6), 1287-1335.

Ceci, F, y Iubatti, D. (2012). Personal relationships and innovation diffusion in SME networks: A content analysis approach. Research Policy, 41(3), 565-579. doi:10.1016/j. respol.2011.10.003

Cho, Y., Hwang, J., y Lee, D. (2012). Identification of effective opinion leaders in the diffusion of technological innovation: A social network approach. Technological Forecasting and Social Change, 79(1), 97-106. doi:10.1016/j. techfore.2011.06.003

Christakis, N. A., y Fowler, J. H. (2008). The collective dynamics of smoking in a large social network.New England journal of medicine, 358(21), 2249-2258.

Cruz, J. P., y Olaya, C. (2008). A system dynamics model for studying the structure of network marketing organizations. In The 2008 International Conference of the System Dynamics Society (pp. 1-34). Athens, Greece.

Delre, S. A., Jager, W., y Bijmolt, T. (2010). Will it spread or not? The effects of social influences and network topology on innovation diffusion. Journal of Product Innovation Management, 27(2), 267-282. doi:10.1111/j.15405885.2010.00714.x

Delre, S. A., Jager, W., Bijmolt, T. H. a., y Janssen, M. A. (2007). Targeting and timing promotional activities: An agent-based model for the takeoff of new products. Journal of Business Research, 60 (8), 826-835. doi:10.1016/j. jbusres.2007.02.002

Euler, Leonhard. "Solutio problematis ad geometriam situs pertinentis" Commentarii academiae scientiarum Petropolitanae 8 (1741): 128-140. 
Fang, X., Hu, P. J.-H., Li, Z., y Tsai, W. (2013). Predicting Adoption Probabilities in Social Networks.Information Systems Research, 24(1), 128-145. doi:10.1287/isre.1120.0461

Garcia, R. (2005). Uses of Agent-Based-Modeling in Innovation / New Product Development Research. Journal of Product Innovation Management, 22(5), 380-398.

Godes, D., y Mayzlin, D. (2004). Using Online Conversations to Study Word-of-Mouth Communication.Marketing Science, 23(4), 545-560. doi:10.1287/mksc. 1040.0071

Goldenberg, J., Han, S., Lehmann, D. R., y Hong, J. W. (2009). The Role of Hubs in the Adoption Process.Journal of Marketing, 73(2), 1-13. doi: 10.1509/jmkg.73.2.1

Goldenberg, J., Libai, B., Moldovan, S., y Muller, E. (2007). The NPV of bad news.International Journal of Research in Marketing, 24(3), 186-200. doi:10.1016/j.ijresmar.2007.02.003

Goldenberg, J., Libai, B., y Muller, E. (2002). Riding the saddle: How cross-market communications can create a major slump in sales. The Journal of Marketing, 66(2), 1-16.

Goldenberg, J., Lowengart, O., y Shapira, D. (2009). Zooming In: Self-Emergence of Movements in New Product Growth.Marketing Science, 28(2), 274-292. doi:10.1287/ mksc. 1080.0395

Granovetter, M. (1973). The strength of weak ties.American journal of sociology, 78, 13601380.

Granovetter, M. (1985). Economic action and social structure: the problem of embeddedness. American Journal of Sociology, 91 (3), 481-510.

Haenlein, M., y Libai, B. (2013a).Targeting Revenue Leaders for a New Product.Journal of Marketing, 77(3), 65-80. doi:10.1509/ jm.11.0428

Hill, S., Provost, F., y Volinsky, C. (2006). Network-Based Marketing: Identifying Likely Adopters via Consumer Networks. Statistical Science, 21(2), 256-276. doi: $10.1214 / 088342306000000222$

Hinz et al. (2012).Seeding Strategies for Viral Marketing: An Empirical Comparison.Journal of Marketing, 75 (6), 55-71.

Iribarren, J. L., y Moro, E. (2011). Affinity Paths and information diffusion in social networks.Social Networks, 33(2), 134-142.

Iyengar, R., Bulte, C. Van den, y Valente, T. W. (2011). Opinion leadership and social contagion in new product diffusion.Marketing Science, 30(2), 195-212.

Janssen, M. A., y Jager, W. (2001). Fashions, habits and changing preferences: Simulation of psychological factors affecting market dynamics. Journal of economic psychology, 22, 745-772.

Katona, Z., Zubcsek, P. P., y Sarvary, M. (2010). Network Effects and Personal Influences: The Diffusion of an Online Social Network. Journal of Marketing Research, 48(3), 425-443.

Kossinets, G., y Watts, D. J. (2006). Empirical analysis of an evolving social network. Science (New York, N.Y.), 311(5757), 88-90. doi:10.1126/science.1116869

Kratzer, J., y Lettl, C. (2009). Distinctive Roles of Lead Users and Opinion Leaders in the Social Networks of Schoolchildren.Journal of Consumer Research, 36(4), 646-659. doi: $10.1086 / 599324$

Laciana, C., Rovere, S., y Podestá, G. (2013). Exploring associations between micro-level models of innovation diffusion and emerging macro-level adoption patterns. Statistical Mechanics and its Applications, 392(8), 18731884.

Libai, B., Bolton, R., Bugel, M. S., de Ruyter, K., Gotz, O., Risselada, H., y Stephen, A. T. (2010). Customer-to-Customer Interactions: Broadening the Scope of Word of Mouth Research. Journal of Service Research, 13(3), 267282. doi:10.1177/1094670510375600

SSN 0122-820X

PP: 28-42 
No. 2

Junio - Diciembre 2013 ISSN 0122-820X

PP: $28-42$
Journal of Marketing Research, 50, 161.

Liu, X., Jiang, T., y Ma, F. (2013). Collective dynamics in knowledge networks: Emerging trends analysis. Journal of Informetrics, 7(2), 425-438. doi:10.1016/j.joi.2013.01.003

Liu-Thompkins, Y. (2012). Seeding Viral Content The Role of Message and Network Factors. Journal of Advertising Research., 59-72. doi: 10.2501/JAR-52-4-000-000

Liu-Thompkins, Y., y Rogerson, M. (2012). Rising to Stardom: An Empirical Investigation of the Diffusion of User-generated Content.Journal of Interactive Marketing, 26(2), 71-82. doi:10.1016/j.intmar.2011.11.003

Mahajan, V., Muller, E., y Bass, F. M. (1990). New product diffusion models in marketing: A review and directions for research. The Journal of Marketing, 54(1), 1-26.

McPherson, M., Smith-Lovin, L., y Cook, J. M. (2001). Birds of a feather: Homophily in social networks. Annual review of sociology, 27, 415-444.

Nitzan, I., y Libai, B. (2011). Social effects on customer retention.Journal of Marketing, 75(April), 1-63.

Pegoretti, G., Rentocchini, F., \&VittucciMarzetti, G. (2012). An agent-based model of innovation diffusion: network structure and coexistence under different information regimes. Journal of Economic Interaction and Coordination, 7(2), 145-165. doi:10.1007/ s11403-012-0087-4

Peres, R., Muller, E., y Mahajan, V. (2010). Innovation diffusion and new product growth models: A critical review and research directions. International Journal of Research in Marketing, 27(2), 91-106. doi:10.1016/j.ijresmar.2009.12.012

Robledo-Giraldo, S., y Osorio-Zuluaga, G. A. (2012).Potencial de ingresos pasivos para los distribuidores en empresas de mercadeo multinivel con un plan de compensación binario. Revista respuestas, 17(2), 13-20.

Rogers. (1962). Diffusion of innovations (Free
Press.). New York.

Rogers, E. M. (2003). Diffusion of Innovations.

Sci2, T. (2009).Science of Science (Sci2) Tool.Indiana University and SciTech Strategies. Retrieved from http://sci2.cns.iu.edu.

Tonta, Y., y Darvish, H. R. (2010). Diffusion of latent semantic analysis as a research tool: A social network analysis approach. Journal of Informetrics, 4(2), 166-174. doi:10.1016/j. joi.2009.11.003

Van den Bulte, C., y Lilien, G. L. (2001). Medical Innovation Revisited: Social Contagion versus Marketing Effort1. American Journal of Sociology, 106(5). R

Van den Bulte, C., y Stremersch, S. (2004). Social contagion and income heterogeneity in new product diffusion: A meta-analytic test. MARKETING SCIENCE, 23(4), 530-544. doi:10.1287/mksc. 1040.0054

Van den Bulte, C., y Yoshi, Y. V. (2007). New product diffusion with influentials and imitators.Marketing Science, 26(3), 400-421. doi:10.1287/mksc.1060.0224

Wasserman, S. (1994). Social Network Analysis: Methods and applications. Vol 8.Cambridge University Press.

Watts, D. J., y Dodds, P. S. (2007). Influentials, Networks, and Public Opinion Formation. Journal of Consumer Research, 34(4), 441-58.

Watts, D. J., y Strogatz, S. H. (1998). Collective dynamics of "small-world" networks. Nature, 393 (6684), 440-2.doi:10.1038/30918

Winkler, W. E. (1990). String Comparator Metrics and Enhanced Decision Rules in the Fellegi-Sunter Model of Record Linkage. Proceedings of the Section on Survey Research Methods (American Statistical Association), 354-359.

Yansong, H. (2013). Hyperlinked actors in the global knowledge communities and diffusion of innovation tools in nascent industrial field. Technovation, 33(2-3), 38-49. doi:10.1016/j. technovation.2012.10.001 